\title{
Efeitos das dimensões da qualidade do serviço na satisfação do usuário do transporte público
}

\author{
Effects of service quality dimensions on public transport user satisfaction
}

\author{
Matheus Grage Tardin ${ }^{1}$, Ariel Barroso da Fonseca ${ }^{2}$, Fábio Reis da Costa ${ }^{3}$, \\ Anderson Soncini Pelissari ${ }^{4}$ \\ ${ }^{1}$ Universidade Federal do Espírito Santo, Espírito Santo - Brasil, mgt091@hotmail.com \\ 2Universidade Federal do Espírito Santo, Espírito Santo - Brasil, digitalmarketing.adm@gmail.com \\ 3Universidade Federal do Espírito Santo, Espírito Santo - Brasil, reis.frc@gmail.com \\ ${ }^{4}$ Universidade Federal do Espírito Santo, Espírito Santo - Brasil, asoncinipelissari@gmail.com
}

\section{Recebido: \\ 27 de abril de 2019 \\ Aceito para publicação: \\ 30 de outubro de 2019 \\ Publicado: \\ 31 de agosto de 2020 \\ Editor de área: \\ Bruno Vieira Bertoncini}

\section{Palavras-chaves:}

Qualidade do serviço.

Transporte público.

P-TRANSQUAL.

Modelagem de equações estruturais.

\section{Keywords:}

Service quality.

Public transport.

P-TRANSQUAL.

Structural equations modeling.

DOI:10.14295/transportes.v28i3.2033

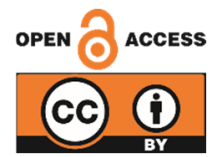

\begin{abstract}
RESUMO
O objetivo deste trabalho é investigar quais atributos da qualidade do serviço do transporte público influenciam na satisfação dos seus usuários. Especificamente, é examinado o efeito das dimensões conforto, tangível, pessoal e confiabilidade na satisfação do usuário do transporte público. A abordagem do estudo é quantitativa, de caráter descritivo e com corte transversal. Os dados empíricos foram coletados por meio de um survey, e a amostra foi composta por 271 respondentes. Para testar as hipóteses teóricas propostas foi empregada a técnica de modelagem de equações estruturais. Os resultados indicam que as dimensões conforto, confiabilidade e tangível possuem efeito positivo e significante na satisfação do usuário do transporte público. Os achados empíricos obtidos neste estudo podem contribuir para a tomada de decisão dos administradores de serviços de transporte público. Entendendo melhor fatores que influenciam a satisfação, torna-se possível o incremento de ações que estimulem a melhoria dos serviços prestados.
\end{abstract}

\section{ABSTRACT}

The aim of this paper is to investigate which attributes of public transport service quality influence the satisfaction of its users. Specifically, it is examined the effect of comfort, tangible, personal and reliability dimensions on public transport user satisfaction. The study approach is quantitative, descriptive and cross-sectional. Empirical data were collected through a survey, with a sample of 271 respondents. To test the proposed theoretical hypotheses, structural equations modeling was applied. The results indicate that comfort, reliability and tangible dimensions have a positive and significant effect on passenger satisfaction. In terms of managerial implications, the empirical findings offer a better understanding of factors that influence public transport passenger satisfaction, so managers can take actions that stimulate and improve the services provided.

\section{INTRODUÇÃO}

O crescimento contínuo das cidades, as questões ambientais, a competição por espaços limitados, e as longas distâncias de deslocamento são as principais razões que tornam o uso do transporte público uma prioridade no Brasil e no mundo (CNT, 2017). 0 transporte público influencia diretamente na qualidade de vida, permitindo a integração, a acessibilidade e a mobilidade da população. Não por acaso o tema é crítico e tem sido discutido em diferentes áreas do conhecimento e setores da sociedade (CNT, 2017; Eboli e Mazzulla, 2007; NTU, 2018). 
No contexto brasileiro, o setor de transporte público coletivo está passando por uma situação difícil. 0 incentivo à aquisição de automóveis e a falta de priorização do transporte público aumentaram os congestionamentos nas áreas urbanas na mesma medida em que elevaram a tarifa e reduziram a qualidade do serviço do transporte público. Nas duas últimas décadas ocorreu uma queda de $44 \%$ na demanda pelo transporte público no Brasil. Somente no período de 2013 a 2017, a perda foi de $25 \%$ (NTU, 2018).

Um aspecto específico dos serviços de transporte público é que eles geralmente são fornecidos por operadores que agem sob contrato com uma autoridade governamental. Em muitos casos, esses serviços são oferecidos por empresas operando em condições quase monopolísticas. Nestas circunstâncias, os operadores do transporte público não estão vinculados a requisitos de mercados competitivos para responder às necessidades dos passageiros, e sim atrelados a cláusulas contratuais.

No entanto, é do interesse de clientes, autoridades, e operadores que os sistemas de transporte público estejam mais focados nas necessidades dos passageiros (Mouwen, 2015). Dessa forma, a fim de assegurar a melhoria contínua do transporte público pela perspectiva do usuário, assim como a prospectar e reter mais passageiros, a satisfação do cliente e a qualidade do serviço se tornam conceitos-chave para gestores e órgãos de trânsito (Eboli e Mazzulla, 2013; Oña e Oña, 2014).

No que diz respeito à relação entre a qualidade do serviço e a satisfação do cliente, pesquisadores (Cronin e Taylor, 1992; Taylor e Baker, 1994; Zeithaml et al., 1996) enfatizam que a qualidade do serviço é considerada um dos antecedentes mais importantes da satisfação do cliente, ou seja, uma qualidade superior do serviço percebido gera um nível mais alto de satisfação do cliente. Tal relação também foi amplamente encontrada no setor do transporte público (Jen et al., 2011; Lai e Chen, 2011; Mugion et al., 2018; Wen et al., 2005).

Apesar da qualidade do serviço ser reconhecida como um construto multidimensional (Grönroos, 1984; Parasuraman et al., 1985; Parasuraman et al., 1988), pesquisas empíricas anteriores que estudaram a relação entre qualidade do serviço e satisfação do consumidor no contexto do transporte público (Jen et al., 2011; Lai e Chen, 2011; Mugion et al., 2018; Wen et al., 2005) não consideraram as dimensões subjacentes da qualidade do serviço em suas análises. Tais pesquisas avaliaram apenas o efeito direto da qualidade geral do serviço na satisfação do usuário.

Embora não haja um consenso quanto à natureza das dimensões da qualidade do serviço, Parasuraman, Zeithaml e Berry (1988) afirmam que os atributos incluídos em uma pesquisa devem ser selecionados para cada caso específico. Assim, este trabalho utilizará o modelo PTRANSQUAL, desenvolvido por Bakti e Sumaedi (2015) especificamente para avaliar a qualidade do serviço de transportes públicos. 0 modelo conta com quatro dimensões: conforto, tangível, pessoal e confiabilidade.

Para abordar esse gap na literatura, surge, então, a seguinte questão de pesquisa: quais atributos da qualidade do serviço do transporte público influenciam na satisfação dos seus usuários? A presente pesquisa tem como objetivo principal identificar os efeitos individuais que as dimensões do modelo P-TRANSQUAL exercem na satisfação do passageiro do transporte público. Como objetivo secundário, este trabalho busca identificar se o sexo e a renda dos passageiros influenciam as relações estudadas.

Ao identificar quais aspectos específicos da qualidade do serviço mais influenciam na satisfação do usuário do transporte público os resultados deste trabalho podem facilitar uma melhor 
alocação de recursos por parte dos operadores do serviço, e dessa forma contribuir para entregar um serviço de melhor qualidade e, consequentemente, gerar um maior nível de satisfação do passageiro. Este trabalho também colabora para este tópico apresentando uma segmentação dos usuários do transporte público. Tal segmentação permite que questões críticas relacionadas aos operadores e ao próprio serviço sejam identificadas dentro de grupos específicos e, assim, apoia decisões direcionadas a cada público-alvo.

Este artigo está organizado da seguinte forma: a seção 2 apresenta o referencial teórico, as hipóteses de pesquisa e o modelo teórico do trabalho; a seção 3 descreve a abordagem metodológica proposta para avaliar quais atributos da qualidade do serviço do transporte público influenciam na satisfação dos seus usuários; a seção 4 apresenta os resultados encontrados; na seção 5 os resultados encontrados são discutidos com base na teoria; por fim a sessão 6 apresenta as conclusões do trabalho.

\section{REFERENCIAL TEÓRICO E HIPÓTESES DE PESQUISA}

Nesta seção são discutidos os conceitos de satisfação do consumidor e de qualidade do serviço no transporte público. Com base na discussão teórica, são formuladas hipóteses de pesquisa sobre a relação entre as dimensões subjacentes da qualidade do serviço e a satisfação do cliente.

\subsection{Qualidade do serviço e satisfação no transporte público}

Qualidade do serviço e satisfação do cliente são construtos conceitualmente distintos, embora intimamente relacionadas (Parasuraman et al., 1988). Na literatura de gestão de serviços, satisfação tem sido comumente definida como a percepção de um indivíduo, como descontentamento ou prazer, ao comparar o desempenho percebido de um serviço em relação às suas expectativas (Kotler e Keller, 2013). Anderson et al. (1994) definiram satisfação como a avaliação global baseada na experiência total de compra e consumo de um bem ou serviço ao longo do tempo. Ou seja, se os clientes avaliarem favoravelmente suas experiências gerais de consumo, é provável que seus níveis de satisfação e de disposição para intenção de recompra aumentem (Chiu et al., 2012).

0 conceito de qualidade de serviço, por outro lado, é complexo, difuso e abstrato, principalmente devido às características intrínsecas aos serviços: intangibilidade, heterogeneidade e inseparabilidade de produção e consumo (Parasuraman et al., 1985). Segundo Zeithaml (1988), a qualidade do serviço é o julgamento do consumidor sobre a excelência ou superioridade geral dos serviços. Portanto, quanto maior o desempenho dos serviços, mais positiva é a percepção do consumidor sobre a qualidade do serviço, e vice-versa. A qualidade do serviço é reconhecida por ser um construto multidimensional (Grönroos, 1984; Parasuraman et al., 1985; Parasuraman et al., 1988).

A literatura aponta que muitos atributos foram usados para avaliar a qualidade do serviço. Murray, Walton e Thomas, (2010), por exemplo, consideraram 166 atributos em seu estudo. Esses atributos são normalmente agrupados em um número menor, chamado de dimensões. Embora não haja consenso quanto à natureza das dimensões da qualidade do serviço, Parasuraman et al. (1988) defenderam a existência de uma lista genérica de atributos e dimensões para analisar a qualidade de qualquer tipo de serviço, o modelo SERVQUAL. Essas dimensões genéricas são: Tangíveis, Confiabilidade, Responsividade, Garantia e Empatia. No entanto, os mesmos autores afirmam que os atributos incluídos em uma pesquisa devem ser selecionados para cada caso específico. 
Pesquisas anteriores que estudaram a relação entre a qualidade do serviço e a satisfação do consumidor encontraram relação positiva e significativa entre os dois construtos (Cronin et al., 2000; Cronin e Taylor, 1992; Parasuraman et al., 1988), inclusive no contexto do transporte público (Abenoza et al., 2017; Jen et al., 2011; Mouwen, 2015).

Poucas pesquisas examinaram a relação entre as dimensões da qualidade do serviço e a satisfação do cliente. Por exemplo, Bowers et al. (1994) descobriram que das 12 dimensões de qualidade de serviço no setor de saúde, apenas seis delas estão significativamente correlacionadas com a satisfação do paciente. No setor bancário, das sete dimensões da qualidade do serviço encontradas por George e Kumar (2014), apenas cinco se mostraram preditoras da satisfação do cliente. E no setor de transporte aéreo, uma das seis dimensões da qualidade do serviço não está correlacionada com a satisfação do cliente (Bezerra e Gomes, 2015).

\subsection{Dimensões da qualidade do serviço no transporte público}

No contexto do transporte público, alguns pesquisadores propuseram modelos de qualidade do serviço considerando características específicas desse tipo de serviço. Por exemplo, Sánchez et al. (2007) modificaram o modelo SERVQUAL e produziram um novo modelo de qualidade de serviço para o serviço de ônibus local, o QUALBUS, que tem cinco dimensões: tangibilidade, confiabilidade, receptividade, garantia e empatia. Lai e Chen (2011) propuseram duas dimensões de qualidade de serviço, que são o serviço principal e o ambiente físico.

Neste trabalho será utilizado o modelo P-TRANSQUAL proposto por Bakti e Sumaedi (2015), que consiste em quatro dimensões: conforto, tangível, pessoal e confiabilidade. Diferente dos outros modelos que são modificações do modelo SERVQUAL, o modelo P-TRANSQUAL foi desenvolvido a partir de extensa revisão de literatura, e foi testado quanto a confiabilidade e validade, atendendo a todos os requisitos de validade discriminante, convergente e relacionada a critério.

Alinhado com o objetivo do trabalho, a seguir será tratada a relação de cada atributo do modelo P-TRASNQUAL com a satisfação do consumidor, para o desenvolvimento das hipóteses de pesquisa.

\subsubsection{Conforto}

A dimensão do conforto é uma dimensão de qualidade de serviço que representa o desempenho no fornecimento de condições confortáveis e de segurança aos passageiros (Bakti e Sumaedi, 2015). Conforto e segurança são dois aspectos inseparáveis do serviço e interconectados. Os passageiros não sentirão conforto antes de se sentirem seguros durante o uso de serviços de transporte público. Em outras palavras, a segurança é um pré-requisito que deve ser cumprido para que os passageiros sintam-se confortáveis em usar o serviço de transporte público (Bakti e Sumaedi, 2015).

Referindo-se ao conceito de classificação de produto de Kotler e Keller (2013), a condição de segurança é o "produto aumentado", enquanto condição confortável é o "produto esperado". No contexto de países desenvolvidos, os provedores de serviços competem no aspecto do produto esperado, enquanto nos países em desenvolvimento, os provedores de serviços geralmente ainda competem no aspecto de produtos aumentados (Kotler e Keller, 2013). Nesse sentido, Parasuraman et al. (1985) definem a dimensão segurança como a ausência da sensação de perigo, risco ou dúvida em relação ao serviço, e pode envolver segurança física, segurança financeira e confidencialidade. 
Estudos empíricos mostraram um efeito positivo da dimensão conforto na satisfação em diversos tipos de serviços, como aeroportuários (Bezerra e Gomes, 2015) e bancários (George e Kumar, 2014). Assim, a primeira hipótese é formulada:

H1: A dimensão conforto tem um efeito positivo na satisfação do usuário de transporte público. 2.2.2. Tangível

Parasuraman et al. (1991) definiram a dimensão tangível como a aparência e condição de instalações físicas, equipamentos, e materiais de comunicação. No contexto do transporte público, a dimensão tangível representa o desempenho do transporte público no aspecto das instalações físicas, a condição do interior, exterior e motor do veículo, abrigo e terminais (Bakti e Sumaedi, 2015).

Se esses tangíveis forem inadequados, indisponíveis ou em más condições, os clientes não se sentirão satisfeitos com o serviço do transporte público. Estudos empíricos mostraram um efeito positivo da dimensão tangível na satisfação em diversos tipos de serviços em companhias aéreas (Leong et al., 2015), hospitais (Calisir et al., 2014), bancos (Yilmaz et al., 2017) e cassinos (Shi et al., 2014). Portanto, a segunda hipótese de pesquisa é formulada:

H2: A dimensão Tangíveis tem um efeito positivo na satisfação do usuário de transporte público.

\subsubsection{Pessoal}

No setor de serviços o ser humano é um aspecto importante para melhorar a qualidade do serviço. Isso ocorre porque o uso (consumo) de um serviço não pode ser separado da interação entre humanos (Parasuraman et al., 1985). Assim, a dimensão Pessoal envolve responsividade, garantia e empatia dos funcionários que mantém contato com o consumidor (Parasuraman et al., 1985). Responsividade pode ser descrita como a disposição de ajudar os clientes e fornecer pronto atendimento, garantia pode ser descrita como o conhecimento e cortesia dos funcionários e sua capacidade de transmitir confiança e segurança, e empatia refere-se a atenção cuidadosa e individualizada fornecida ao cliente (Parasuraman et al., 1991).

No contexto do transporte público, algumas interações ocorrem frequentemente entre os passageiros e os funcionários, como a notificação do destino, problemas ou reclamação relatados, e o pagamento dos serviços (Bakti e Sumaedi, 2015). Estudos empíricos mostraram um efeito positivo da dimensão pessoal na satisfação em diversos tipos de serviços, como bancário (Sharma, 2015) e hospitais (Meesala e Paul, 2016). Assim, a terceira hipótese de pesquisa é formulada:

H3: A dimensão Pessoal tem um efeito positivo na satisfação do usuário de transporte público.

\subsubsection{Confiabilidade}

Cada serviço tem um benefício central que se torna o principal objetivo da prestação de serviços (Kotler e Keller, 2013). Nesse sentido a Confiabilidade é descrita como a capacidade de executar o serviço prometido de maneira confiável e precisa (Parasuraman et al., 1991). No contexto dos serviços de transporte público, o benefício principal é levar os passageiros ao destino (Bakti e Sumaedi, 2015). Assim, alguns aspectos importantes que devem ser considerados na dimensão da confiabilidade, como a quantidade de veículos, o tempo de espera, o tempo de viagem e a consistência dos serviços no transporte dos passageiros até o destino (Bakti e Sumaedi, 2015).

Se o nível de confiabilidade for alto espera-se que os clientes fiquem satisfeitos com o serviço prestado. Estudos empíricos mostraram um efeito positivo da dimensão confiabilidade na satisfação em diversos tipos de serviços, como companhias aéreas (Leong et al., 2015), hospitais 
(Calisir et al., 2014), bancário (George e Kumar, 2014), cassinos (Shi et al., 2014), entre outros. Portanto, a quarta hipótese proposta é:

H4: A dimensão Confiabilidade tem um efeito positivo na satisfação do usuário de transporte público.

\subsection{Modelo teórico da pesquisa}

Com base na revisão da literatura e considerando as hipóteses da pesquisa, o modelo da Figura 1 será testado no estudo.

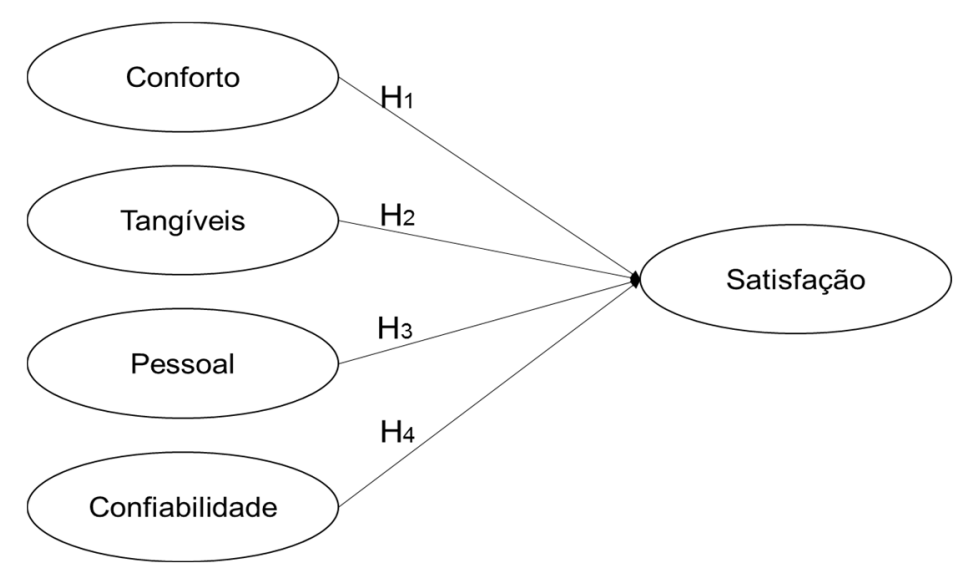

Figura 1. Modelo teórico da pesquisa

\section{ASPECTOS METODOLÓGICOS DA INVESTIGAÇÃO}

Para alcançar o objetivo proposto, bem como testar as hipóteses de pesquisa, a abordagem metodológica deste trabalho foi quantitativa, do tipo levantamento, e com corte transversal. A proposta do levantamento é descrever as características de certo fenômeno ou população e estabelecer relações entre variáveis (Creswell, 2010).

Para testar o modelo teórico da pesquisa, foram analisados os dois maiores sistemas de transportes públicos de ônibus do estado do Espírito Santo: o Sistema Municipal de Transporte de Vitória e o Sistema Transcol. O Sistema Municipal de Transporte de Vitória é composto por 56 linhas de ônibus distribuídas em 342 veículos, que possuem idade média de 4,5 anos. 0 sistema transporta por dia aproximadamente 120 mil passageiros (Vitória, 2016).

o Sistema Transcol é um sistema metropolitano integrado de estrutura tronco-alimentadora, que interliga os cinco municípios da Região Metropolitana da Grande Vitória (Vitória, Serra, Vila Velha, Cariacica e Viana) por meio de dez terminais urbanos. 0 sistema conta com 1445 ônibus com idade média de 6,66 anos, e atendeu em 2018 mais de 170 milhões de passageiros (CETURB-ES, 2018).

A população selecionada para este estudo foram os estudantes da Universidade Federal do Espírito Santo, campus Goiabeiras. 0 campus foi selecionado por estar situado na área urbana da cidade de Vitória, e por ser servido por diversas linhas do transporte público municipal e intermunicipal. Ademais, os estudantes do Campus são provenientes dos diferentes municípios da Grande Vitória e muitos utilizam o transporte público como meio de locomoção e acesso à Universidade. 
0 estudo foi realizado em seis etapas distintas. Inicialmente o questionário foi desenvolvido a partir da operacionalização das variáveis. Em seguida foi realizado um pré-teste para analisar a aplicabilidade do questionário em relação ao seu entendimento e formato. Na terceira etapa ocorreu a coleta dos dados da pesquisa. Na quarta etapa foi realizada a avaliação do modelo de mensuração, para avaliar o nível em que a escala realmente mensura o que ela pretende medir (Hair et al., 2014). Em seguida, o modelo estrutural foi analisado por meio de modelagem de equações estruturais (PLS-SEM) (Hair et al., 2014; Sanchez, 2013). Por fim, os dados da pesquisa foram estratificados entre os sexos, renda e entre o transporte público municipal e intermunicipal, e a diferença foi testada usando análise multi-grupo (PLS-MGA) (Sarstedt e Ringle, 2011).

A Modelagem de Equações Estruturais (PLS-SEM) utiliza um conjunto de métodos estatísticos multivariados para identificar e analisar múltiplas relações de dependência entre variáveis. Um de seus principais usos é identificar quais variáveis mais influenciam outra variável (Hair et al., 2014), como é o caso deste trabalho. Ademais, por se tratar de um método não-paramétrico, o PLS-SEM não assume que os dados sejam normalmente distribuídos. Assim, para testar a significância dos resultados, o algoritmo do PLS-SEM realiza um procedimento conhecido como bootstrap (Sanchez, 2013).

No bootstrap muitas amostras são criadas a partir de observações retiradas aleatoriamente do conjunto original de dados (com substituição), sendo que cada amostra possui o mesmo número de observações que a amostra original. As amostras são então usadas para estimar o modelo de caminho PLS, ou seja, ao usar 5.000 sub amostras, 5.000 modelos PLS diferentes são calculados. As estimativas dos coeficientes desses modelos formam uma distribuição de bootstrap. Com base nessa distribuição é possível determinar o erro padrão e o desvio padrão dos coeficientes estimados, e assim testar sua significância (Hair et al., 2014; Sanchez, 2013).

Para a análise dos dados foi utilizado o software R (R Core Team, 2018) e o pacote "plspm" (Sanchez, 2013).

\subsection{Operacionalização das variáveis}

Esta pesquisa estuda cinco variáveis latentes distintas: conforto, tangíveis, pessoal, confiabilidade e satisfação do usuário. Para mensurar as variáveis, o instrumento de pesquisa foi elaborado com base nos estudos de Bakti e Sumaedi (2015) e Sumaedi et al. (2016). As variáveis e seus respectivos indicadores são apresentados na Tabela 1.

Seguindo o estudo de Bakti e Sumaedi (2015), os entrevistados foram solicitados a expressar sua percepção sobre 18 afirmações positivas sobre o indicador de qualidade de serviço do transporte público e sobre quatro indicadores de satisfação (Sumaedi et al., 2016). A escala do questionário foi de sete pontos na escala Likert, onde 1 representa "discordo totalmente" e 7 representa "concordo totalmente".

Além das variáveis de pesquisa, o questionário também foi composto por duas questões controle e duas questões socioeconômicas. A primeira questão-controle foi feita para garantir que o respondente tenha de fato utilizado o serviço de ônibus, enquanto a segunda questão-controle questionou qual dos dois sistemas o respondente utilizou. Para identificar o perfil do respondente, foram questionados o sexo e a renda familiar. Dessa forma, o instrumento de pesquisa foi composto por 26 questões. 
Tabela 1 - Variáveis e indicadores

\begin{tabular}{ll}
\hline & \multicolumn{1}{c}{ Indicador } \\
\hline Conforto & Segurança relacionada ao crime \\
CO1 & Temperatura confortável \\
CO2 & Segurança ao usar serviços de transporte público \\
CO3 & Segurança relacionada à violência e assédio \\
CO4 & Capacidade de passageiros \\
CO5 & Obediência às leis de trânsito \\
CO6 & \\
Tangíveis & Limpeza do interior, assentos e janelas do veículo \\
TA1 & Limpeza do exterior do veículo \\
TA2 & Condição dos assentos \\
TA3 & Condição da máquina (motor) \\
TA4 & Responsividade \\
Pessoal & Utilidade dos funcionários \\
PE1 & Compreensão da necessidade dos passageiros \\
PE2 & Cortesia de pessoal \\
PE3 & \\
PE4 & Tempo de espera \\
Confiabilidade \\
CE1 & Adequação às necessidades dos passageiros \\
CE2 & Tempo de viagem \\
CE3 & Chegada ao destino \\
CE4 & \\
Satisfação & O serviço percebido foi equivalente ao serviço ideal \\
SA1 & A viagem foi prazerosa \\
SA2 & No geral, fiquei satisfeito com o transporte público \\
SA3 & O serviço percebido excedeu minhas expectativas \\
SA4 &
\end{tabular}

\subsection{Amostra e coleta dos dados}

Inicialmente, foi realizado um teste-piloto com 30 universitários para avaliar o questionário em termos de redação, clareza, relevância, e tempo gasto. Após pequenos ajustes, o questionário foi encaminhado via sistema interno aos alunos da universidade no período de julho de 2018. Foram obtidas 271 respostas. Após a eliminação dos respondentes que não utilizam transporte público, a amostra final foi composta por 233 usuários do transporte público. A Tabela 2 resume o perfil da amostra.

No que tange o tamanho e validade da amostra, o PLS-SEM possui poucos requisitos quando comparado com outras técnicas estatísticas. 0 tamanho mínimo de amostra deve ser dez vezes o maior número de caminhos estruturais direcionados a uma determinada variável no modelo estrutural (Hair et al., 2014). Como a variável satisfação recebe quatro caminhos, a amostra mínima deve ser de 40 respondentes. A amostra também excede o requisito para pesquisas de marketing definido por Malhotra (2001), de 200 respondentes, e é maior que a de estudos anteriores, como o de Mugion et al. (2018) cuja amostra foi de 114 respondentes

Tabela 2 - Perfil dos respondentes usuários do transporte público

\begin{tabular}{lll}
\hline Variáveis & & $\%$ \\
\hline Sexo & Feminino & 52 \\
& Masculino & 48 \\
Renda familiar & $<R \$ 1.908,00$ & 25 \\
& $\mathrm{R} \$ 1.908,00-\mathrm{R} \$ 3.816,00$ & 38 \\
& $\mathrm{R} \$ 3.816,00-\mathrm{R} \$ 9.540,00$ & 30 \\
& $>\mathrm{R} \$ 9.540,00$ & 7 \\
Tipo de transporte & Municipal & 25 \\
& Intermunicipal & 75 \\
\hline
\end{tabular}




\section{RESULTADOS}

Após a coleta, os dados foram analisados seguindo as etapas propostas por Hair et al. (2014) e Sanchez (2013) para modelagem de equações estruturais. Inicialmente foi analisado o modelo de mensuração e em seguida o modelo estrutural. A análise do modelo de mensuração tem como objetivo verificar a qualidade das escalas utilizadas para medir as variáveis do modelo. A análise do modelo estrutural, por sua vez, objetiva examinar a capacidade preditiva do modelo e os relacionamentos entre as variáveis.

\subsection{Análise do modelo de mensuração}

Nessa etapa dois aspectos são avaliados: a validade convergente e a validade discriminante do modelo. A validade convergente descreve a capacidade dos indicadores de medir uma variável, enquanto a validade discriminante descreve o quanto uma variável e seus indicadores são diferentes das outras variáveis e seus respectivos indicadores (Hair et al., 2014; Sanchez, 2013).

Quatro critérios foram utilizados para avaliar a validade convergente (Hair et al., 2014; Sanchez, 2013). O primeiro foi o Cronbach's Alpha, que calcula a correlação média entre os indicadores de um mesmo construto. Se um bloco de indicadores mede uma mesma variável, eles devem ser altamente correlacionados. Como regra geral, o Cronbach's Alpha maior que 0,6 é considerado aceitável. 0 segundo critério foi o Dillon-Goldstein rho que mede o quanto a variável latente explica seu bloco de indicadores. Em regra geral, um bloco é considerado como unidimensional quando o Dillon-Goldstein rho é maior que 0,7 .

Tabela 3 - Validade convergente

\begin{tabular}{|c|c|c|c|c|c|}
\hline \multicolumn{2}{|c|}{ Indicador } & $\begin{array}{l}\text { Alfa de } \\
\text { Cronbach }\end{array}$ & DG.rho & AVE & Carga \\
\hline \multicolumn{2}{|c|}{ Conforto } & 0.817 & 0.880 & 0.65 & \\
\hline $\mathrm{CO} 1$ & Segurança relacionada ao crime & & & & 0.845 \\
\hline $\mathrm{CO} 2$ & Temperatura confortável & & & & 0.749 \\
\hline $\mathrm{CO} 3$ & Segurança ao usar serviços de transporte público & & & & 0.790 \\
\hline $\mathrm{CO} 4$ & Segurança relacionada à violência e assédio & & & & 0.827 \\
\hline \multicolumn{2}{|c|}{ Tangíveis } & 0.844 & 0.897 & 0.67 & \\
\hline TA1 & Limpeza do interior, assentos e janelas do veículo & & & & 0.842 \\
\hline TA2 & Limpeza do exterior do veículo & & & & 0.784 \\
\hline TA3 & Condição dos assentos & & & & 0.781 \\
\hline TA4 & Condição da máquina (motor) & & & & 0.884 \\
\hline \multicolumn{2}{|c|}{ Pessoal } & 0.934 & 0.953 & 0.83 & \\
\hline PE1 & Responsividade & & & & 0.926 \\
\hline PE2 & Utilidade dos funcionários & & & & 0.873 \\
\hline PE3 & Compreensão da necessidade dos passageiros & & & & 0.920 \\
\hline PE4 & Cortesia de pessoal & & & & 0.930 \\
\hline \multicolumn{2}{|c|}{ Confiabilidade } & 0.836 & 0.890 & 0.66 & \\
\hline CE1 & Tempo de espera & & & & 0.830 \\
\hline CE2 & Adequação às necessidades dos passageiros & & & & 0.817 \\
\hline CE3 & Tempo de viagem & & & & 0.810 \\
\hline CE4 & Chegada ao destino & & & & 0.814 \\
\hline \multicolumn{2}{|c|}{ Satisfação } & 0.898 & 0.930 & 0.78 & \\
\hline SA1 & O serviço percebido foi equivalente ao serviço ideal & & & & 0.908 \\
\hline SA2 & A viagem foi prazerosa & & & & 0.903 \\
\hline SA3 & No geral, fiquei satisfeito com o transporte público & & & & 0.914 \\
\hline SA4 & O serviço percebido excedeu minhas expectativas & & & & 0.773 \\
\hline
\end{tabular}

O próximo critério foi examinar as cargas dos fatores. As cargas são as correlações entre uma variável e cada um de seus indicadores, e por meio dela é possível verificar se os indicadores em um bloco são bem explicados pela variável latente. Cargas maiores que 0,7 são aceitáveis. 
O último critério utilizado para verificar a validade convergente foi a variância média extraída (AVE). Este critério é definido como o valor médio das cargas dos indicadores associados a uma variável, elevado ao quadrado. Um valor de AVE igual ou superior a 0,5 é aceitável.

Dois indicadores da dimensão conforto, "capacidade de passageiros" e "obediência às leis de trânsito", não passaram no critério da carga dos fatores por apresentarem os valores 0,622 e 0,588, respectivamente. Como a retirada dos indicadores gerou aumento no Cronbach's Alpha, no Dillon-Goldstein rho, AVE, e nas cargas dos demais fatores, os dois indicadores foram eliminados do modelo de mensuração. Conforme apresentado na Tabela 3, após a eliminação todas variáveis e indicadores passaram nos critérios da validade convergente.

Para avaliar a validade discriminante Hair et al. (2014) recomenda o uso do critério de Fornell-Lacker, no qual a raiz quadrada da AVE de cada variável deve ser maior que a sua correlação com qualquer outra variável. A Tabela 4, apresenta os resultados do teste.

Tabela 4 - Validade discriminante critério de Fornell-Lacker

\begin{tabular}{llllll}
\hline & Conforto & Tangíveis & Pessoal & Confiabilidade & Satisfação \\
\hline Conforto & $\mathbf{0 . 8 0 4}$ & & & & \\
Tangíveis & 0.542 & $\mathbf{0 . 8 2 4}$ & & & \\
Pessoal & 0.475 & 0.416 & $\mathbf{0 . 9 1 3}$ & & \\
Confiabilidade & 0.603 & 0.523 & 0.500 & $\mathbf{0 . 8 1 8}$ & \\
Satisfação & 0.722 & 0.563 & 0.447 & 0.706 & $\mathbf{0 . 8 7 7}$ \\
\hline
\end{tabular}

A raiz quadrada do AVE dos construtos, em negrito na Tabela 4, é maior do que a correlação entre os construtos. Dessa forma todas as variáveis passaram no teste de Fornell-Lacker. Após a análise e consequente ajuste do modelo de mensuração, a próxima etapa foi avaliar o modelo estrutural.

\subsection{Avaliação do modelo estrutural}

A análise dos resultados do modelo estrutural seguiu três passos (Hair et al., 2014). 0 primeiro foi analisar o grau de colinearidade entre as variáveis latentes independentes do modelo, o segundo passo foi avaliar o valor do coeficiente de determinação $\left(\mathrm{R}^{2}\right)$, e o terceiro foi avaliar a significância e relevância dos coeficientes de caminho.

Para avaliar a colinearidade, Hair et al. (2014) sugere utilizar o fator de inflação da variância, conhecido como VIF. Se o nível de colinearidade for muito alto (VIF $\geq 5$ ), deve-se considerar a remoção de um dos indicadores correspondentes. Conforme apresentado na Tabela 5, todos os construtos passam no teste.

Tabela 5 - Avaliação da colinearidade

\begin{tabular}{lllll}
\hline & Conforto & Tangível & Pessoal & Confiabilidade \\
\hline VIF Satisfação & 2.711 & 2.446 & 1.486 & 2.462 \\
\hline
\end{tabular}

O próximo passo foi avaliar o coeficiente de determinação $\left(R^{2}\right) .0 R^{2}$ é a medida mais comumente usada para avaliar o modelo estrutural. Esse coeficiente é a medida de apuração preditiva do modelo, representando os efeitos combinados das variáveis independentes na variável dependente (Hair et al., 2014). 
$\mathrm{O}$ valor de $\mathrm{R}^{2}$ varia de 0 a 1 , com valores mais próximos a 1 indicando níveis mais altos de precisão preditiva. Em pesquisas acadêmicas que focam em questões de marketing, os valores de $\mathrm{R}^{2}$ de $0,75,0,50$ ou 0,25 podem, como regra geral, ser respectivamente descritos como substanciais, moderados ou fracos (Hair et al., 2014). Os resultados encontrados revelam um $\mathrm{R}^{2} \mathrm{da}$ variável satisfação de 0,649 , ou seja, $64,9 \%$ da variação da satisfação é explicada pela variação das variáveis independentes. 0 modelo, portanto, possui apuração preditiva moderada.

Para medir a contribuição de cada construto independente para o $\mathrm{R}^{2}$ do construto dependente, é necessário calcular os valores do tamanho do efeito $\left(\mathrm{f}^{2}\right)$. Hair et al. (2014) recomendam que os valores do $\mathrm{f}^{2}$ de 0,35, 0,15 e 0,02 devem ser considerados grandes, médios e pequenos, respectivamente. A contribuição das dimensões conforto e confiabilidade para $o \mathrm{R}^{2}$ da satisfação está no nível médio-forte enquanto a contribuição da dimensão tangíveis é fraca. A dimensão pessoal, por sua vez, não contribui para o $\mathrm{R}^{2}$ da satisfação. A Tabela 6 resume os resultados do teste $\mathrm{f}^{2}$.

Tabela 6 - Tamanho do efeito $\mathrm{f}^{2}$

\begin{tabular}{llll}
\hline & $\mathbf{R}^{\mathbf{2}}$ incluso & $\mathbf{R}^{\mathbf{2}}$ excluído & $\mathbf{f}^{\mathbf{2}}$ \\
\hline Conforto -> Satisfação & 0.649 & 0.559 & 0.256 \\
Tangíveis -> Satisfação & 0.649 & 0.637 & 0.034 \\
Pessoal -> Satisfação & 0.649 & 0.649 & 0.000 \\
Confiabilidade -> Satisfação & 0.649 & 0.568 & 0.231 \\
\hline
\end{tabular}

Por fim, o próximo passo foi avaliar os coeficientes de caminho. Os valores de um coeficiente de caminho variam entre -1 e +1 , quanto mais próximas de +1 representam uma relação forte $\mathrm{e}$ positiva, e vice e versa para valores negativos. Quanto mais próximas as estimativas forem de 0 mais fraca é a relação, sendo usualmente não significante (Hair et al., 2014). A determinação de significância de um coeficiente é dada a partir da análise do erro padrão obtido pela validação bootstrapping (Hair et al., 2014). Assim, foi realizada uma validação bootstrap com 5000 sub amostras (nível de significância $=0,05$ ) para avaliar a significância dos coeficientes de caminho. Os resultados estão apresentados na Tabela 7.

Tabela 7 - Relevância e significância dos coeficientes de caminho

\begin{tabular}{lcl}
\hline Caminho & Coeficiente de caminho & p-Valor \\
\hline Conforto -> Satisfação & 0.418 & 0.000 \\
Tangíveis -> Satisfação & 0.136 & 0.000 \\
Pessoal -> Satisfação & 0.000 & 0.998 \\
Confiabilidade -> Satisfação & 0.384 & 0.000 \\
\hline
\end{tabular}

Os resultados mostram que os valores dos coeficientes de caminho das variáveis conforto, tangíveis e confiabilidade à variável satisfação são significantes e relevantes. 0 coeficiente de caminho da dimensão pessoal à satisfação se mostrou insignificante e irrelevante, não sendo assim um fator determinante da satisfação dos passageiros.

A partir da análise do modelo estrutural, especificamente da relevância e significância dos coeficientes de caminho, as hipóteses de pesquisa foram avaliadas. As hipóteses de pesquisa H1, H2 e H4 são suportadas. 0 impacto da dimensão pessoal na satisfação se revelou insignificante, não sendo possível assim suportar a hipótese de pesquisa H3. 


\subsection{Análise multi-grupo}

Hair et al. (2014) alerta que a heterogeneidade é frequente em pesquisas empíricas e Sanchez (2013) explica que a análise dos coeficientes de caminhos é insuficiente quando diferentes grupos de uma mesma amostra apresentam heterogeneidade. Nesse sentido, os dados da pesquisa foram estratificados entre os sexos, renda e o transporte público municipal e intermunicipal. 0 mesmo algoritmo PLS com validação bootstrap foi utilizado para calcular os coeficientes de caminho para cada sexo, renda e tipo de transporte. A diferença entre os caminhos foi testada usando análise multi-grupo (PLS-MGA) (Sarstedt e Ringle, 2011).

Inicialmente a amostra foi estratificada entre os sexos masculino e feminino. A comparação dos coeficientes de caminho mostrou que não há diferença significativa entre os sexos. Para realizar a análise multi-grupo da renda, os respondentes foram divididos em dois grupos: renda alta e renda baixa. 0 primeiro grupo foi composto por quem tem renda familiar acima de quatro salários mínimos ( $\mathrm{R} \$ 3.992,00)$, enquanto o segundo grupo foi composto pelos respondentes com renda de até quatro salários mínimos. Os resultados revelam que também não há diferença significativa entre os coeficientes de caminho dos dois grupos.

Por fim, foi realizada a análise multi-grupo entre os dois sistemas de transporte públicos por ônibus analisados, o Municipal de Vitória e o Transcol. Os resultados mostram que não existe diferença significativa entre os dois grupos para as dimensões conforto, tangíveis e confiabilidade. Entretanto, para o coeficiente de caminho entre a dimensão pessoal e a satisfação, os resultados apontam que existe diferença significativa entre os dois sistemas. Para o Municipal de Vitória o coeficiente de caminho foi negativo $(-0,158)$, enquanto para o Transcol foi positivo $(0,056)$.

\section{DISCUSSÃO}

Os resultados revelaram que a dimensão conforto é o preditor mais importante e tem o efeito mais forte na satisfação. A dimensão do conforto é uma dimensão que representa o desempenho no fornecimento de condições confortáveis e de segurança aos passageiros (Bakti e Sumaedi, 2015). O resultado está em concordância com estudos anteriores (Bezerra e Gomes, 2015; George e Kumar, 2014) que também encontraram efeito positivo e significante entre conforto e satisfação. Portanto, os gestores do transporte público que desejam aumentar a satisfação do usuário precisam garantir que boas condições de conforto e segurança sejam uma prioridade. É somente com um ambiente seguro e confortável que os clientes ficarão satisfeitos com sua jornada. Isso pode ser alcançado com melhorias na segurança, tanto relacionada ao crime quanto à violência e assédio, e garantindo uma temperatura confortável no interior do veículo, por exemplo.

O estudo também mostrou que a dimensão confiabilidade é o segundo mais importante preditor da satisfação, assim como o segundo maior efeito direto. A confiabilidade é descrita como a capacidade de executar o serviço prometido de maneira confiável e precisa (Parasuraman et al., 1991). No contexto dos serviços de transporte público, o benefício principal é levar os passageiros ao destino (Bakti e Sumaedi, 2015). A literatura aponta que medidas que melhoram a regularidade do transporte público têm efeitos positivos adicionais na confiabilidade do serviço (Jenelius, 2018). Nesse sentido, gestores precisam garantir que o serviço seja sempre confiável. Redução do tempo de espera e do tempo de viagem, assim como entender e adequar o serviço 
às necessidades dos passageiros são fatores que podem aumentar a satisfação do usuário do transporte público.

A dimensão tangível também tem um impacto e poder de predição significativo na satisfação, porém esse impacto é muito pequeno em comparação com os do conforto e da confiabilidade. Essa dimensão representa o desempenho do transporte público no aspecto das instalações físicas, a condição do interior, exterior e motor do veículo, abrigo e terminais (Bakti e Sumaedi, 2015; Parasuraman et al., 1991). 0 resultado encontrado está de acordo com os estudos de Calisir et al. (2014) e Leong et al. (2015), que também encontraram relação positiva e significativa entre as variáveis. Gestores do transporte público devem se manter atentos às condições físicas dos veículos, instalações e equipamentos.

Diferentemente das demais dimensões, o impacto e a capacidade de predição da dimensão pessoal em relação à satisfação foram insignificantes e irrelevantes. A dimensão pessoal se refere a responsividade, garantia e empatia dos funcionários que mantém contato com o consumidor (Parasuraman et al., 1985). Esse resultado é contrário aos de Sharma (2015) e Meesala e Paul (2016), mas em consonância com Leong et al. (2015). 0 impacto insignificante está relacionado à percepção dos clientes de que os funcionários dos sistemas de transporte público analisados são responsivos, compreensivos e corteses, mas que ainda assim estão insatisfeitos com o serviço. Dessa forma, os passageiros não consideram a dimensão pessoal como um fator determinante da satisfação.

A segmentação pelo sexo e pela renda não apontou diferenças significativas entre os grupos analisados. Dessa forma é possível afirmar que a população estudada não apresenta heterogeneidade entre os sexos e entre respondentes de renda alta e baixa. Ou seja, os coeficientes de caminho entre as dimensões da qualidade do serviço e a satisfação do consumidor são estatisticamente iguais para os sexos masculino e feminino, assim como para respondentes de alta e baixa renda.

A comparação dos coeficientes de caminho dos respondentes que utilizam o Sistema Municipal de Vitória e os que utilizam os Sistema Transcol, apontou diferença significativa apenas para o construto pessoal. Esse resultado mostra que, apesar de serem administrados por diferentes empresas e serem regulados por diferentes agências governamentais, não existem diferenças significativas para a relação entre as dimensões confiabilidade, tangíveis, e conforto e a satisfação do consumidor entre os dois sistemas.

\section{CONCLUSÃO}

Esta pesquisa teve por objetivo identificar quais atributos da qualidade do serviço do transporte público influenciam na satisfação dos seus usuários. Dessa forma, foram analisados a relação entre as dimensões do modelo P-TRANSQUAL (Bakti e Sumaedi, 2015) - confiabilidade, tangível, pessoal, e conforto - com a satisfação de passageiros universitários de dois sistemas de transporte público brasileiros. Para alcançar o objetivo proposto, foram levantadas quatro hipóteses de pesquisa e foi desenvolvido um modelo teórico. 0 modelo foi analisado por meio de modelagem de equações estruturais.

Os resultados da análise do modelo teórico apontaram para a sustentação das hipóteses de pesquisa 1, 2, e 4, e para a rejeição da hipótese 3. Assim, em resposta ao objetivo do trabalho, os atributos da qualidade do serviço do transporte público que influenciam na satisfação do passageiro são conforto, confiabilidade e tangíveis. A dimensão conforto é a que exerce a maior 
influência e tem a maior capacidade preditiva da satisfação, seguido pela confiabilidade. A dimensão tangível, quando comparado com o conforto e a confiabilidade, exerce um pequeno efeito, e tem uma pequena capacidade de predição da satisfação.

Ações de melhoria na qualidade do serviço podem levar a uma maior satisfação do usuário do transporte público, principalmente as relacionadas à segurança e conforto, e à confiabilidade. É somente com um ambiente seguro e confortável e com um serviço confiável que os clientes ficarão satisfeitos com sua jornada. Reduzir a insegurança ao utilizar o transporte público, assim como o tempo de espera e de viagem, são ações que devem ser consideradas pelos gestores do transporte público.

Em termos de implicações gerenciais, os achados empíricos obtidos neste estudo podem contribuir para a tomada de decisão dos administradores de serviços de transporte público. Entendendo melhor os fatores que influenciam a satisfação, tornando-se possível o incremento de ações que estimulem a melhoria dos serviços prestados. Como a dimensão conforto se mostrou o mais importante percussor da satisfação, é necessário um esforço extra dos gestores para garantir que os passageiros se sintam seguros e confortáveis na utilização do transporte público. Prover um serviço confiável também está entre os fatores mais importantes a serem tratados. Devido à importância do transporte público no dia-a-dia dos usuários, qualquer atraso ou falha resultará em insatisfação. Assim, melhorar os processos internos para garantir um serviço confiável deve estar entre as principais preocupações dos gestores do transporte público.

Teoricamente, esta pesquisa foi capaz de avançar na literatura referente ao impacto das dimensões da qualidade do serviço na satisfação do usuário do setor do transporte público. A pesquisa é uma das primeiras a examinar o efeito simultâneo das dimensões da qualidade do serviço do modelo P-TRANSQUAL - conforto, tangível, pessoal e confiabilidade - na satisfação do usuário do serviço do transporte público. Assim, o modelo conceitual deste estudo pode ser usado como fonte de referência para outros pesquisadores interessados em estudar o impacto das dimensões da qualidade do serviço na satisfação do usuário do setor do transporte público.

Dentre as limitações deste artigo, destacam-se o foco na população universitária, a escolha do modelo de qualidade do serviço do transporte público, e ao fato de que a investigação foi realizada em apenas uma cidade brasileira e em apenas dois sistemas de transporte. Assim, como sugestão de pesquisas futuras, sugere-se a investigação das relações das dimensões da qualidade do serviço no transporte público com a satisfação do passageiro em outros contextos culturais, em um maior número de cidades, assim como o estudo com população para além dos estudantes universitários. Sugere-se também a escolha de outros modelos de qualidade do serviço e a realização da pesquisa com dados longitudinais.

\section{AGRADECIMENTOS}

O presente trabalho foi realizado com apoio da Coordenação de Aperfeiçoamento de Pessoal de Nível Superior - Brasil (CAPES) - Código de Financiamento 001.

\section{REFERÊNCIAS}

Abenoza, R. F.; O. Cats e Y. O. Susilo (2017) Travel satisfaction with public transport: Determinants, user classes, regional disparities and their evolution. Transportation Research Part A: Policy and Practice, v. 95, p. 64-84. DOI:10.1016/j.tra.2016.11.011

Anderson, E. W.; C. Fornell e D. R. Lehmann (1994) Customer Satisfaction, Market Share, and Profitability: Findings from Sweden. Journal of Marketing, v. 58, n. 3. DOI:10.2307/1252310

Bakti, I. e S. Sumaedi (2015) P-TRANSQUAL: a service quality model of public land transport services. International Journal of Quality \& Reliability Management, v. 32, n. 6, p. 534-558. DOI: 10.1108/MRR-09-2015-0216 
Bezerra, G. C. L. e C. F. Gomes (2015) The effects of service quality dimensions and passenger characteristics on passenger's overall satisfaction with an airport. Journal of Air Transport Management, v. 44-45, p. 77-81. DOI:10.1016/j.jairtraman.2015.03.001

Calisir, F; A. Bayraktaroglu; C. Gumussoy e B. Kaya (2014) Effects of service quality dimensions including usability on perceived overall quality, customer satisfaction, and return intention in different hospital types. Int. J. Advanced Operations Management, v. 6, n. 4, p. 309-323. DOI: 10.1504/IJAOM.2014.066829

CETURB-ES (2018) Dados Operacionais. Obtido de https://ceturb.es.gov.br/dados-operacionais

Chiu, C.; M. Hsu; H. Lai e C. Chang (2012) Re-examining the in fluence of trust on online repeat purchase intention: The moderating role of habit and its antecedents. Decision Support Systems, v. 53, n. 4, p. 835-845. DOI:10.1016/j.dss.2012.05.021

CNT. (2017) Pesquisa Mobilidade da População Urbana 2017. Brasilia.

Creswell, J. W. (2010) Projeto de Pesquisa: métodos qualitativos, quantitativo e misto. (3. ed.). Artmed, Porto Alegre.

Cronin, J. J.; M. K. Brady e G. T. M. Hult (2000) Assessing the Effects of Quality, Value, and Customer Satisfaction on Consumer Behavioral Intentions in Service Environments. Journal of Retailing, v. 76, n. 2, p. 193-218. DOI: 10.1016/S00224359(00)00028-2

Cronin, J. J. e S. A. Taylor (1992) Measuring Quality: A Reexamination and Extension. Journal of Marketing, v. 56, n. 3, p. 55-68. DOI:10.2307/1252296

Eboli, L. e G. Mazzulla (2007) Service Quality Attributes Affecting Customer Satisfaction for Bus Transit. Journal of Public Transportation, v. 10 n. 3, p. 21-34. DOI:10.5038/2375-0901.10.3.2

Eboli, L. e G. Mazzulla (2013) Performance indicators for an objective measure of public transport service quality. European Transport, v. 51, p. 1-21.

George, A. e G. S. G. Kumar (2014) Impact of service quality dimensions in internet banking on customer satisfaction. DECISION, v. 41, n. 1, p. 73-85. DOI:10.1007/s40622-014-0028-2

Grönroos, C. (1984) A Service Quality Model and its Marketing Implications. European Journal of Marketing, v. 18, n. 4, p. 3644. DOI:10.1108/MBE-09-2016-0047

Hair, J. F.; G. T. M. Hult; C. M. Ringle e M. S. Rstedt (2014) A Primer on Partial Least Squares Structural Equation Modeling (PLSSEM). Sage Publications, Thousand Oaks, California.

Jen, W.; R. Tu e T. Lu (2011) Managing passenger behavioral intention: An integrated framework for service quality, satisfaction, perceived value, and switching barriers. Transportation, v. 38, n. 2, p. 321-342. DOI:10.1007/s11116-010-9306-9

Jenelius, E. (2018) Public transport experienced service reliability : Integrating travel time and travel conditions. Transportation Research Part A, v. 117, p. 275-291. DOI:10.1016/j.tra.2018.08.026

Kotler, P. e K. L. Keller (2013) Marketing management. Prentice Hall.

Lai, W. T. e C. F. Chen (2011) Behavioral intentions of public transit passengers-The roles of service quality, perceived value, satisfaction and involvement. Transport Policy, v. 18, n. 2, p. 318-325. DOI:10.1016/j.tranpol.2010.09.003

Leong, L.; T. Hew; V. Lee e K. Ooi (2015) An SEM - artificial-neural-network analysis of the relationships between SERVPERF, customer satisfaction and loyalty among low-cost and full-service airline. Expert systems with applications, v. 42, n. 19. DOI:10.1016/j.eswa.2015.04.043

Meesala, A. e J. Paul (2016) Service quality , consumer satisfaction and loyalty in hospitals : Thinking for the future. Journal of Retailing and Consumer Services, v. 40, p. 261-269. DOI:10.1016/j.jretconser.2016.10.011

Mouwen, A. (2015) Drivers of customer satisfaction with public transport services. transportation research part A, v. 78, p. 120. DOI:10.1016/j.tra.2015.05.005

Mugion, R. G.; M. Toni; H. Raharjo; L. Di Pietro e S. P. Sebathu (2018) Does the service quality of urban public transport enhance sustainable mobility? Journal of Cleaner Production, v. 174, p. 1566-1587. DOI:10.1016/j.jclepro.2017.11.052

Murray, S. J.; D. Walton e J. A. Thomas (2010) Attitudes towards public transport in New Zealand. Transportation, v. 37, n. 6, p. 915-929. DOI:10.1007/s11116-010-9303-z

NTU (2018) Anuário NTU 2017-2018. Brasilia.

Oña, J. e R. Oña (2014) Quality of Service in Public Transport Based on Customer Satisfaction Surveys : A Review and Assessment of Methodological Approaches. Transportation Science, Articles in Advance, v. 49, n3, p. 1-18.

DOI: $10.1287 /$ trsc.2014.0544

Parasuraman, A.; L. Berry e V. A. Zeithaml (1991) Perceived service quality as a customer-based performance measure: An empirical examination of organizational barriers using an extended service quality model. Human Resource Management, v. 30, n. 3, p. 335-364. DOI:10.1002/hrm.3930300304

Parasuraman, A.; V. Zeithaml e L. L. Berry (1985) A Conceptual Model of Service Quality and Its Implications for Future Research. American Marketing Association, v. 49, n. 4, p. 41-50. DOI:10.2307/1251430

Parasuraman, A.; V. Zeithaml e L. L. Berry (1988) SERVQUAL: A Multiple Item Scale for Measuring Consumer Perceptions of Service Quality. Journal of Retailing, v. 64, n. 1, p. 12-37. D0I:10.1016/S0148-2963(99)00084-3

Sanchez, G. (2013) PLS Path Modeling with R. Trowchez Editions, Berkeley.

Sánchez, M.; J. Carlos; G. María e R. Sánchez (2007) Effects of service quality dimensions on behavioural purchase intentions. Managing Service Quality: An International Journal, v. 17, n. 2, p. 134-151. DOI:10.1108/09604520710735164

Sarstedt, M. e C. M. Ringle (2011) Multigroup Analysis in Partial Least Squares (PLS) Path Modeling : Alternative Methods and Empirical Results. Advances in International Marketing, v. 22, p. 195-218.

DOI:10.1108/S1474-7979(2011)0000022012 
Sharma, V. (2015) International Journal of Bank Marketing. International Journal of Bank Marketing, v. 33, n. 4. DOI:10.1108/IJBM-04-2014-0048

Shi, Y.; C. Prentice e W. He (2014) Linking service quality, customer satisfaction and loyalty in casinos: does membership matter? International Journal of Hospitality Management, v. 40, p. 81-91. DOI:10.1016/j.ijhm.2014.03.013

Sumaedi, S.; I. Bakti; T. Rakhmawati; N. J. Astrini; T. Widianti e M. Yarmen (2016) Factors influencing public transport passengers' satisfaction: a new model. Management of Environmental Quality: An International Journal, v. 27, n. 5, p. 585-597. DOI:10.1108/MEQ-05-2015-0084

Taylor, S. A. e T. L. Baker (1994) An assessment of the relationship between service quality and customer satisfaction in the formation of consumers' purchase intentions. Journal of Retailing, v. 70, n. 2, p. 163-178. DOI:10.1016/00224359(94)90013-2

Vitória. (2016) A Cidade: Transporte coletivo. Obtido de http://www.vitoria.es.gov.br/cidade/transporte-coletivo

Wen, C. H.; L. W. Lan e H. L. Cheng (2005) Structural Equation Modeling to Determine Passenger Loyalty Toward Intercity Bus Services. Journal of Transportation Research Board, n. 1927, p. 249-255.

DOI:10.3141/1927-28

Yilmaz, V.; E. Ari e H. Gurbuz (2017) Investigating the relationship between service quality dimensions, customer satisfaction and loyalty in Turkish banking sector: an application of structural equation model. International Journal of Bank Marketing, v. 36, n. 3, p. 423-440. DOI: 10.1108/IJBM-02-2017-0037

Zeithaml, V. A. (1988) Consumer Perceptions of Price, Quality, and Value: A Means-End Model and Synthesis of Evidence. Journal of Marketing, v. 52, n. 3, p. 2-22. DOI:10.2307/1251446

Zeithaml, V. A.; L. L. Berry e A. Parasuraman (1996) The Behavioral Consequences of Service Quality. Journal of Marketing, v. 60, p. 31-46. DOI: $10.2307 / 1251929$ 